\title{
Harnblasenkarzinom: Blaulichtzystoskopie verbessert Diagnose und Therapie
}

\begin{abstract}
— Das Harnblasenkarzinom ist der vierthäufigste Tumor des Mannes. Unabhängig vom Tumorstadium zur Zeit der Diagnose, ist bei allen Patienten mit nicht muskelinvasivem Harnblasenkarzinom (NMIBC) eine lange Beobachtungszeit auch nach Resektion erforderlich, da ein hohes Risiko für lokale Rezidive besteht. Je nach Erstbefund rezidiviert ein Blasenkarzinom bei über der Hälfte der Patienten innerhalb von fünf Jahren, da bei der Resektion residuales Tumorrestgewebe oder mikroskopische Läsionen übersehen werden. Die photodynamische Diagnose mit Hexaminolevulinate (HAL, Hexvix ${ }^{\oplus}$ ) in Verbindung mit Weißlicht (WL) macht mehr Läsionen sichtbar.
\end{abstract}

Laut einer auf dem Kongress der European Association of Urology (EAU) 2013 vorgestellten Metaanalyse lässt sich mit der Blaulichtzystoskopie das Rezidiv-Risiko senken [Burger et al. EAU 2013, Abstract 577]. Prof. Christian G. Stief, München, kommentierte im Interview: „Die bessere Detektion von Harnblasenkarzinomen ermöglicht eine wesentlich sicherere Resektion der betroffenen Blasentumore, was mit deutlich verminderten Rezidivraten einhergeht."

Mit HAL wurden $22 \%$ mehr Ta/T1-Läsionen entdeckt, die mit alleinigem Weißlicht nicht gesehen wurden. Auch $24 \%$ der Insitu-Tumoren wurden mit Blaulicht-, nicht aber durch alleinige Weißlichtzystoskopie, entdeckt. Die suboptimale Visualisierung des Blasentumors mit Weißlicht führt, wie die Metaanalyse bestätigt, häufiger zu einem frühen Rezidiv. Die Rezidivrate nach 9-12 Monaten war bei HAL-Technik mit $34,5 \%$ vs. $45,4 \%$ signifikant geringer ( $R R=$ $0,761 ; p=0,006)$. „Ich denke, dass wir auch eine Verringerung der Progressionsrate sehen werden, aber dafür brauchen wir eine längere Nachbeobachtungszeit", so Stief.

Auf der neuen Internetplatform www. innovators-in-bc.com finden Urologen und Onkologen ein internationales, wissenschaftsorientiertes Forum im Kampf gegen das Harnblasenkarzinom.

Dr. Ine Schmale

"Media meets the expert" im Rahmen des Kongresses der European Association of Urology 2013, Mailand, 17. März 2013, mit Unterstützung von Ipsen Pharma, Ettlingen

\section{Dauertherapie bei BPH/LUTS: weniger Prostatasymptome, bessere Erektionsfähigkeit}

— Für Männer mit benigner Prostatahyperplasie (BPH) und Symptomen der unteren Harnwege (Lower Urinary Tract Symptoms, LUTS), die keiner Operation bedürfen, stehen bei Vorliegen einer erektilen Dysfunktion (ED) verschiedene medikamentöse Optionen zur Verfügung. Bei Tadalafil (Cialis ${ }^{\circledast)}$ könnten sich Ärzte auf umfangreiche Daten stützen, sagte der Urologe Dr. Sidney Glina, São Paulo, Brasilien. Komfortabel für

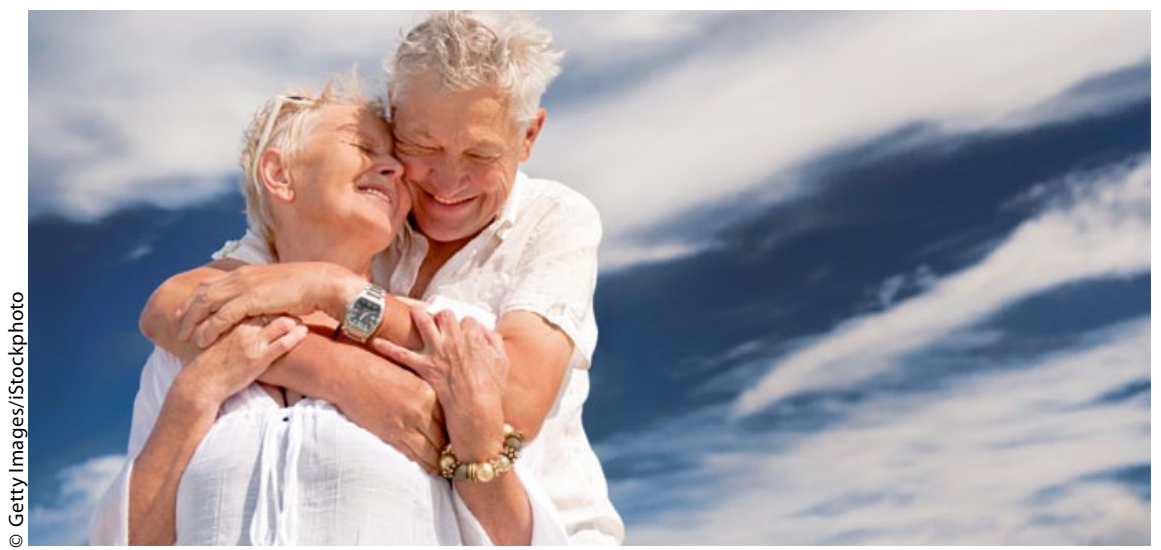

Bei Patienten mit benigner Prostatahyperplasie (BPH) und erektiler Dysfunktion kann die medikamentöse Therapie neben der Erektionsfähigkeit auch die BPH-assoziierten Symptome bessern.

Patienten bei einer Dauertherapie sei die einmal tägliche Einnahme.

In einer Phase-III-Studie mit rund 600 sexuell aktiven Männern mit ED und BPH/LUTS bewirkte die zwölfwöchige Einnahme von einmal täglich $5 \mathrm{~g}$ Tadalafil verglichen mit Placebo signifikante Verbesserungen im Internationalen Prostata-Symptomen-Score (IPSS) sowie der Erektionsfähigkeit $(\mathrm{p}<0,001)$ [Egerdie RB et al. J Sex Med 2012; 9: 271-81].
In einer weiteren Studie war Tadalafil Tamsulosin 0,4 mg hinsichtlich der Verbesserungen im IPSS über zwölf Wochen nicht unterlegen [Oelke $M$ et al. Eur Urol 2012; 61: 917-25]. Verglichen mit Placebo steigerte es - im Unterschied zu Tamsulosin - auch die Erektionsfähigkeit $(p<0,001)$. In einer offenen Extensionsstudie blieb die Wirksamkeit von Tadalafil auf die BPH-assoziierten Symptome ein Jahr lang erhalten [Donatucci CF et al. BJU Int 2011; 107: 1110-6]. Die Kombination von Tadalafil $5 \mathrm{mg}$ plus Finasterid $5 \mathrm{mg}$ vs. Finasterid/Placebo prüften Glina et al. in einer Studie mit 695 Männern mit symptomatischer BPH. Im IPSS bewirkte die Kombination signifikant stärkere Verbesserungen als Finasterid/Placebo $(p<0,05)$ [International Symposium on Prostate, Androgens and Men's Sexual Health, Berlin, 2013, Abstract 21-003]. Die Kombination steigerte nicht nur bei sexuell aktiven Männern mit ED die Erektionsfähigkeit $(p<0,001)$. Auch bei jenen ohne Erektionsprobleme zu Studienbeginn ergaben sich signifikante Verbesserungen gegenüber der Finasterid-Monotherapie $(p<0,05)$.

Dr. Matthias Herrmann

Round-Table „Evidence based Medicine Treatment of Patients with Prostate and Sexual Problems - Tricky Cases from the Expert's Practice"; International Symposium on Prostate, Androgens and Men's Sexual Health der European Society for Sexual Medicine und der International Society for Sexual Medicine, Berlin, 21.Juni 2013 Veranstalter: Lilly, Bad Homburg 